\title{
Le tenaci malte della torre del castello di Cerreto Ciampoli (Siena, Italia)
}

Strong mortars from the tower of the Cerreto Ciampoli castle (Siena, Italy)

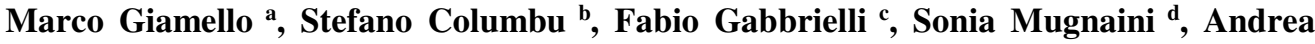 \\ Scala $^{\mathrm{e}}$ \\ a Dipartimento di Scienze Fisiche, della Terra e dell'Ambiente - Università di Siena, Siena, Italy, marco.giamello@unisi.it \\ b Dipartimento di Scienze Chimiche e Geologiche - Università di Cagliari, Cagliari, Italy, columbus@unica.it \\ ${ }^{\mathrm{c}}$ Dipartimento di Scienze Storiche e dei Beni Culturali - Università di Siena, Siena, Italy, fabio.gabbrielli@unisi.it \\ ${ }^{d}$ Dipartimento di Scienze Fisiche, della Terra e dell'Ambiente - Università di Siena, Siena, Italy, mugnaini12@unisi.it \\ ${ }^{\text {e }}$ Dipartimento di Scienze Fisiche, della Terra e dell'Ambiente - Università di Siena, Siena, Italy, andrea.scala@ unisi.it
}

\begin{abstract}
Known since the eleventh century, the castle of Cerreto Ciampoli was one of the main fortifications of the ancient Republic of Siena (Tuscany, Italy). The magnificent ruins, located on the top of a hill overlooking the Chianti Mountains, consist of two city walls, a door, a church, the remains of some rooms and a mighty tower lying on the ground broken up into five sections of several meters in length. The present study is focused on the analysis of the mineralogical-petrographic and chemical features of the sack and the bedding mortars of the tower, and it is aimed at understanding the exceptional qualities of these mortars that, during the collapse of the artifact, prevented the tower from shattering into smaller pieces. The tenacity of these mortars appears to be the result of the concurrence of more expedients, such as the choice of well-selected materials (hydraulic limes obtained from the local Alberese limestone, sandy aggregates from well-rinsed river sands with a high silicoclastic component) and the use of particular technical methods (i.e. hot lime technique).
\end{abstract}

Keywords: Chianti Mountains, Cerreto Ciampoli castle, Republic of Siena, historical mortars, applied petrography.

\section{Introduzione}

Il presente studio ha come oggetto le malte della torre del castello di Cerreto Ciampoli (Comune di Castelnuovo Berardenga, Siena, Italia). Tale manufatto fa parte di un più ampio complesso fortificato di accertata importanza storica e strategica per il territorio in questione. La torre si presenta oggi sotto forma di cinque tronconi di notevoli dimensioni adagiati a terra, in ognuno dei quali le malte, che caratterizzano sia il nucleo della muratura che l'allettamento di bozze in pietra e di laterizi del paramento, si contraddi- stinguono per un grado di adesione e di coesione particolarmente elevato.

Com'è noto, l'analisi mineralogico-petrografica e chimica delle malte storiche è particolarmente utile nella identificazione della natura e della provenienza delle materie prime e nel riconoscimento delle relative tecnologie di produzione (e.g. Cantisani, et al., 2018; Chiarelli, et al., 2015; Franzini, et al., 2000; Giamello, et al., 2017; Guasparri, et al., 2006; Lezzerini, et al., 2017; Moropoulou, et al., 2000; Riccardi, et al., 
2007). Attraverso tale approccio si intende raccogliere elementi utili per l'identificazione di materiali e procedimenti tecnologici che hanno contribuito alla fabbricazione delle malte di eccezionali qualità della torre in oggetto, tanto da garantire la durevolezza della muratura fino ai nostri giorni ed impedire, in occasione del crollo, la frantumazione della struttura in elementi minuti.

\section{Nota storica e descrizione dell'apparato murario}

Il castello di Cerreto (Fig. 1), detto anche Cerreto Ciampoli o Cerretaccio, località attestata fin dal 1097, costituì il principale fortilizio della potente consorteria senese dei Cerretani. Tra la fine del Duecento e il primo Quattrocento fu acquisito, in più tempi, dalla Repubblica di Siena di cui divenne uno dei capisaldi della linea di confine con il territorio controllato da Firenze (Cammarosano, Passeri, 1985; Fusai, 2010).

Lavori di fortificazione e di riparazione del castello sono documentati negli anni 1229-1230, 1268, 1398 e 1401-1402 (Passeri, 2002). Nel 1491, a conferma del ruolo strategico del sito, il Comune di Siena incaricò alcuni maestri, tra $\mathrm{i}$ quali l'architetto senese Francesco di Giorgio Martini, a ricostruire la fortezza, ma non è chiaro cosa effettivamente sia stato realizzato (Adams, 1993; Chironi, 1993).

Grandiose rovine, riferibili a diverse fasi edilizie, testimoniano l'importanza del castello e la sua continuità fino alla caduta dello Stato senese, avvenuta alla metà del XVI secolo (Gabbrielli, 1996; Marchetti, 2016). Sulla cima di una boscosa collina prospiciente i Monti del Chianti sono infatti riconoscibili due cinte murarie, una porta, i resti di una seconda porta, una chiesa dai caratteri tardoromanici e i resti di alcuni ambienti di incerta funzione. La struttura più spettacolare è una poderosa torre, a pianta rettangolare con base di circa sette metri di lato (misure interne: $2.42 \times 2.86 \mathrm{~m}$ ), la quale, caduta a terra forse a seguito di una deliberata opera di abbattimento (Parenti, Doglioni, 1993; Valenti, 1995), si presenta, eccezionalmente, spezzata in più tronconi, il primo dei quali raggiunge circa i 7.5 metri di lunghezza. I muri, che alla base misurano oltre due metri di spessore, presentano un paramento a bozze in pietra squadrate e un nucleo in calcestruzzo ad altissimo grado di adesione e coesione. La parte sommitale della struttura era in mattoni, come si evince da due tronconi ubicati nella parte terminale del crollo.

Il rivestimento della torre è realizzato principalmente in pietra Alberese, un calcare marnoso di colore variabile dal biancastro al grigio chiaro al nocciola solcato da sottili vene di calcite spatica. Tale litotipo deriva dai depositi flyschoidi della Formazione di Monte Morello di età eocenica, che affiora estesamente nel territorio in oggetto, in particolare tra Firenze e le colline del Chianti (Lazzarotto, et al., in stampa), e costituisce il substrato su cui insiste il castello stesso. Dal punto di vista petrografico l'Alberese si classifica come una biomicrite, con contenuto fossilifero rappresentato da radiolari, spicole di spugne e foraminiferi planctonici. Studi riferiti all'area fiorentina (Fratini, et al., 1994) mostrano un contenuto di $\mathrm{CaCO}_{3}$ ampiamente variabile dal 55 al $90 \%$, a seconda del livello stratigrafico e del sito. La frazione non carbonatica è rappresentata da minerali argillosi (caolinite, illite, clorite, illite-smectite e clorite-vermiculite) e quantità minori di quarzo e feldspati. Il calcare Alberese (denominato "albazzano" già nei trattati quattrocenteschi; Martini, 1967) è storicamente utilizzato in Toscana sia come pietra da costruzione tal quale che come pietra da calce (Fratini, et al., 1994; Pecchioni, et al., 2006).

Le malte di allettamento e del nucleo, assai tenaci e compatte, mostrano un colore variabile da avana a grigiastro chiaro. Quelle di allettamento sono caratterizzate da aggregati con granuli di dimensioni per lo più fino a millimetriche, mentre in quelle del nucleo si osservano comunemente frammenti anche centimetrici. Sono inoltre presenti abbondanti grumi di dimensioni variabili da qualche millimetro fino a vari centimetri, e relitti di cottura dell'originaria pietra da calce di colore bianco e nocciola. Abbondanti concrezioni calcaree derivate da addensamento del legante sono visibili in più parti della muratura sia del nucleo che del paramento esterno. 

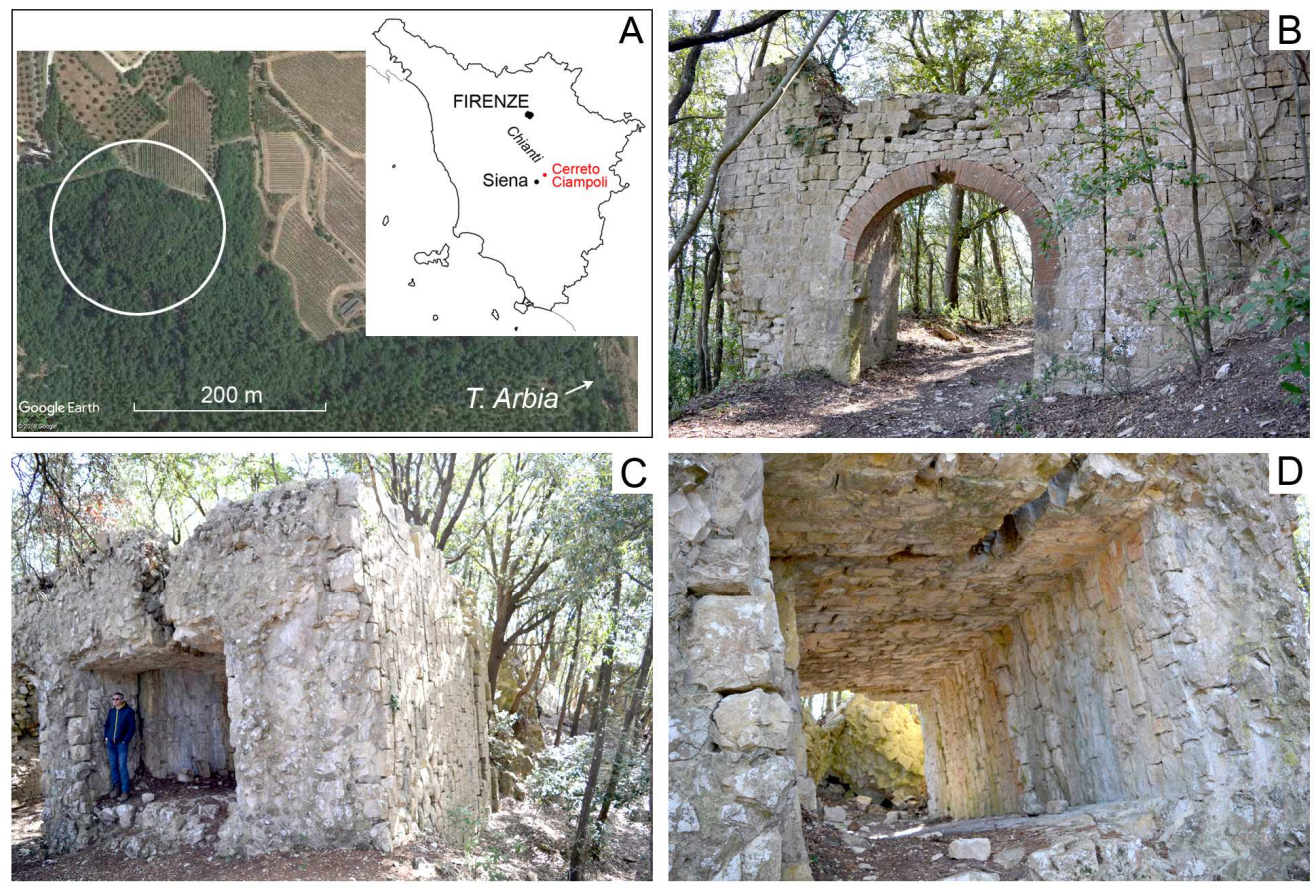

Fig. 1. Ruderi del castello di Cerreto Ciampoli. A) Vista della zona dall'alto (cerchio bianco); B) Ingresso del castello; C) Troncone principale della torre; D) Interno della torre.

\section{Materiali e metodologie analitiche adottate}

Sono stati prelevati cinque campioni di malta dai giunti tra le bozze squadrate del paramento e dal nucleo della torre, sia nelle parti in pietra che in laterizi (Tab. 1). Ogni campione consiste in un unico frammento di dimensioni pluricentimetriche (Fig. 2).

\begin{tabular}{ccc}
\hline $\begin{array}{c}\text { N. } \\
\text { camp. }\end{array}$ & Tipo e ubicazione & Colore \\
\hline 1 & Troncone 1, MA & H9,3YR;V7,3;C1,9 \\
2 & Troncone 1, MN & H9,2YR;V7,2;C1,8 \\
3 & Troncone 2, MA & H9,6YR;V7,0;C1,7 \\
4 & Troncone 3, MN & H9,3YR;V6,9;C2,1 \\
5 & Troncone 4, MA & H9,5YR;V6,9;C2,2 \\
\hline
\end{tabular}

Tab. 1. Campioni di malte prelevate dalla torre del castello di Cerreto Ciampoli. MA: malta di allettamento; $\mathrm{MN}$ : malta del nucleo. Il colore è espresso secondo la notazione di Munsell.
Sui campioni di malta prelevati è stata effettuata preliminarmente un'osservazione macroscopica e allo stereomicroscopio e, a valle di una blanda pulitura, sono state effettuate le misurazioni del colore mediante il colorimetro Minolta Chroma Meters CR 200. I risultati sono riportati nello spazio dei colori di Munsell.

Successivamente sono state preparate due o più sezioni sottili per ogni campione e analizzate mediante microscopio ottico polarizzatore. Sono state eseguite analisi modali per la quantificazione dei componenti della malta, conteggiando 300 punti per ogni campione in porzioni rappresentative, con un passo di $1 \mathrm{~mm}$ nelle due dimensioni. I granuli di dimensioni eccedenti 1 $\mathrm{mm}$ sono stati conteggiati una sola volta. Dall'analisi modale si è ottenuta anche una prima stima sulla distribuzione dimensionale dei granuli e sui rapporti legante/aggregato.

Le analisi in microscopia ottica sono state supportate da diffrattometria a raggi $\mathrm{X}$ delle polveri (XRPD). Lo strumento impiegato è un diffrattometro Philips X'Pert PRO PW 3040 a 

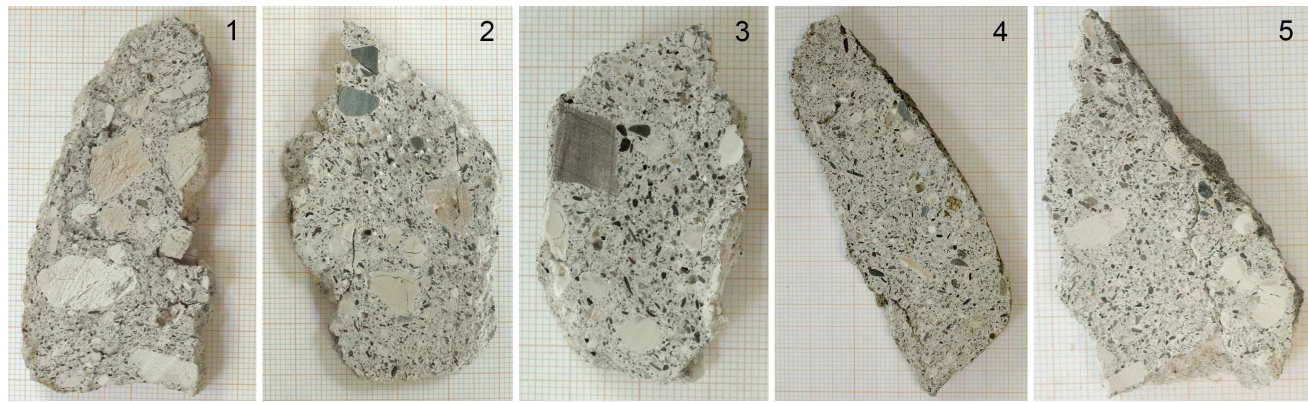

Fig. 2. Aspetto macroscopico delle malte oggetto di indagine.

geometria Bragg-Brentano equipaggiato di detector PW3015 X'Celerator, usato alle condizioni operative di $40 \mathrm{kV}$ e $40 \mathrm{~mA}$ e impiegando la radiazione $\mathrm{CuK} \alpha$. Gli spettri sono stati raccolti nell'intervallo $3-60^{\circ}(2 \theta)$ con una velocità di scansione di $0,016 \%$ s.

Per una migliore caratterizzazione del legante, sono state eseguite analisi chimiche sui grumi. Per ogni campione, numerosi grumi sono stati isolati sotto controllo allo stereomicroscopio al fine di allontanare eventuali componenti dell'aggregato. Dal materiale derivante, finemente macinato, sono stati prelevati $100 \mathrm{mg}$ di polvere, i quali sono stati riscaldati con $\mathrm{Li}_{2} \mathrm{~B}_{4} \mathrm{O}_{7}$ e $\mathrm{LiBO}_{2}$ a $950^{\circ} \mathrm{C}$ per 30 minuti in crogioli di platino (fusione alcalina).

La perla vetrosa è stata solubilizzata con una soluzione al 5\% di $\mathrm{HNO}_{3}$. Le analisi sono state effettuate con uno spettrofotometro ad emissione ottica con sorgente ICP (ICP-OES) PerkinElmer Optima 2000. L'accuratezza delle analisi è stata verificata usando gli standard internazionali JLs-1 (calcare, GSJ) e GBW07108 (roccia carbonatica, GBW). La precisione analitica, determinata come \%RSD su cinque repliche, è sempre risultata entro il $5 \%$ per concentrazioni superiori a $0.1 \%$ (in ossidi). L'indice di idraulicità (I.I.) viene espresso come I.I. $=\left(\mathrm{SiO}_{2}+\right.$ $\left.\mathrm{Al}_{2} \mathrm{O}_{3}+\mathrm{Fe}_{2} \mathrm{O}_{3}\right) /(\mathrm{CaO}+\mathrm{MgO})$.

\section{Risultati}

Dall'analisi mineralogico-petrografica i materiali provenienti dai tronconi della torre sono risultati molto simili fra loro (Fig. 3). Si tratta di malte che presentano un legante a base di calce e un aggregato prevalentemente sabbioso. Il rapporto legante/aggregato risulta per lo più compreso tra $1 / 2$ e $1 / 2,5$. L'aggregato è relativamente ben sortito, con una distribuzione granulometrica unimodale non molto allargata in cui prevale la componente di sabbia fine-sabbia media. I granuli mostrano principalmente una sfericità medio-alta e una morfologia da sub-angolosa ad arrotondata. Dal punto di vista composizionale, l'aggregato è costituito da monocristalli, frammenti litici, rari microfossili carbonatici e rari frammenti di terracotta. I granuli di quarzo sono presenti sia come monocristalli che come aggregati policristallini (con cristalli sia deformati che non deformati). I grani con composizione carbonatica sono rappresentati da frammenti di micrite/microsparite (tra cui calcari marnosi), monocristalli e aggregati policristallini di calcite spatica e assai rari microfossili (gusci di bivalvi e foraminiferi). I feldspati sono presenti come monocristalli di plagioclasio (con composizione di tipo oligoclasio-albite e albite) e di kfeldspato (ortoclasio e microclino). Le miche sono rappresentate da lamelle e aggregati policristallini di muscovite e/o biotite, quest'ultima talora alterata in clorite. I frammenti litici sono rappresentati principalmente da arenaria quarzoso-feldspatica con matrice argillosa, argillite, siltite e selce. Tra i componenti rari e accessori (riportati come "altri componenti" in Fig. 3) vi sono granuli costituiti da associazioni di quarzo e feldspati derivati da rocce magmatiche intrusive, frammenti di micascisto, frammenti di serpentinite, minerali opachi, monocristalli di zircone e di titanite. 

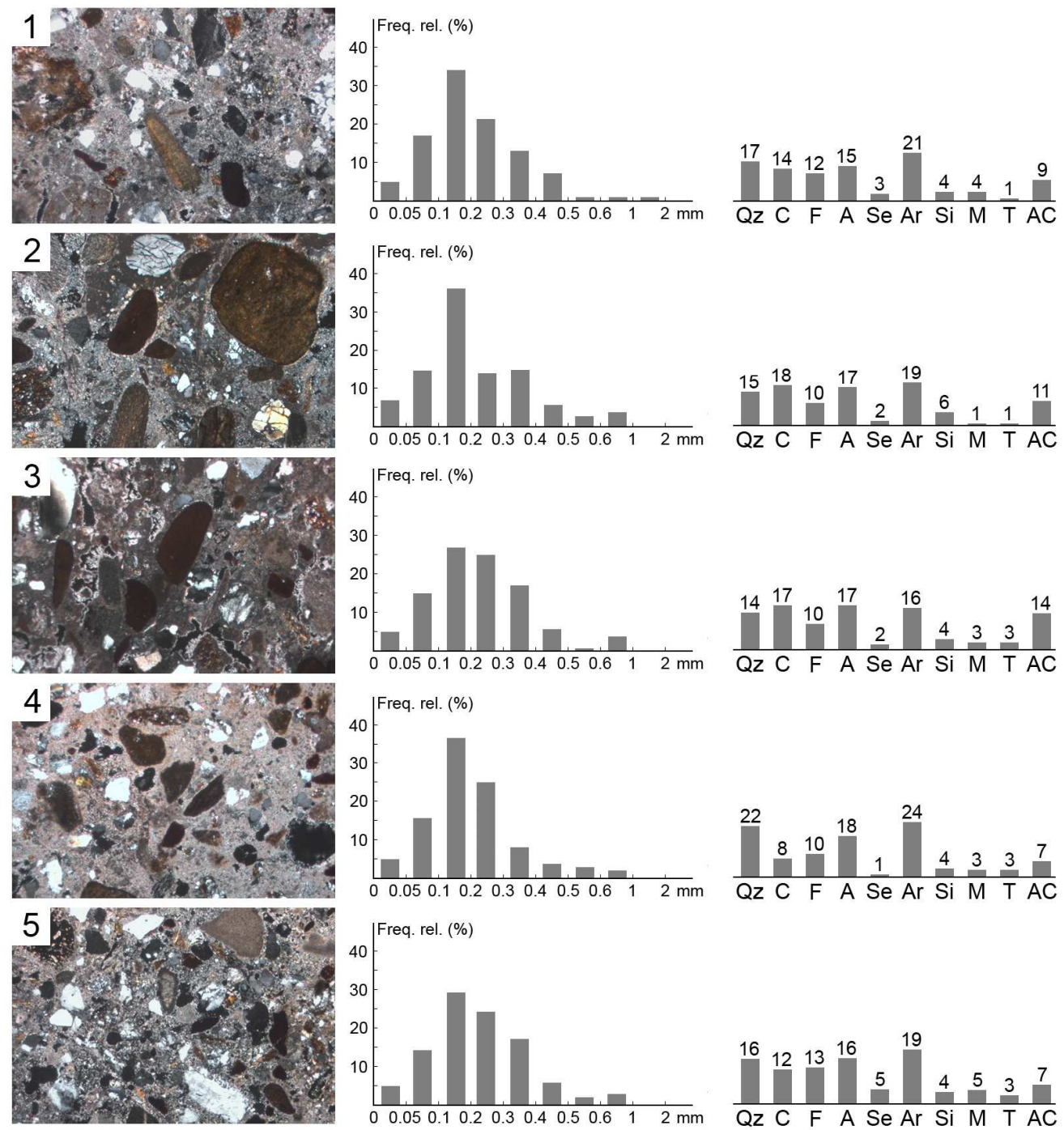

Fig. 3. Sintesi dei risultati dell'analisi petrografica degli aggregati delle malte oggetto di indagine. A sinistra: immagini ottenute al microscopio ottico polarizzatore in sezione sottile, Nicol incrociati. La base di ciascuna foto corrisponde a $4 \mathrm{~mm}$. Al centro: distribuzione dimensionale dei granuli dell'aggregato nell'intervallo $0-2 \mathrm{~mm}$. A destra: risultati dell'analisi modale, con indicazione della percentuale dei vari componenti (Qz: monocristalli e aggregati policristallini di quarzo; C: granuli a composizione carbonatica; F: feldspati; A: arenaria; Se: selce; Ar: argillite; Si: siltite; M: miche; T: terracotta; AC: altri componenti).

Il legante mostra una struttura micro-criptocristallina. Sono presenti, in quantità variabili, grumi di calce a tessitura omogenea, di dimensioni da qualche decina di $\mu \mathrm{m}$ fino a quelli pluricentimetrici visibili ad occhio nudo. I frammenti incotti della originaria pietra da calce, scarsa- mente riconoscibili, mostrano una tessitura microcristallina con venature assai sottili.

La porosità è generalmente bassa in tutti i campioni analizzati ed è rappresentata da pori con forme irregolari variamente associati a calcite di riprecipitazione secondaria. 
Le analisi chimiche dei grumi (Tab. 2) mostrano l'uso di calci ad elevata idraulicità, con variabile ma sostanziale presenza delle componenti idrauliche $\mathrm{SiO}_{2}, \mathrm{Al}_{2} \mathrm{O}_{3}$ e $\mathrm{Fe}_{2} \mathrm{O}_{3}$ e valori di I.I. anche più elevati rispetto a quelli tipici delle normali calci idrauliche (da 0,40 a 1,10 ).

\section{Discussione e conclusioni}

L'insieme dei dati ottenuti consente di fare alcune considerazioni in merito alla tenacia mostrata dalle malte della torre del castello di Cerreto Ciampoli. Si ritiene che questa sia il risultato del concorso di più fattori che riguardano sia le specifiche materie prime impiegate (tipologie di calce e di aggregato), sia la tecnica di preparazione dell'impasto.

Per quanto riguarda la calce, si tratta di una calce avente caratteristiche fortemente idrauliche derivanti dalla cottura di un calcare marnoso o di una marna. Le composizioni rilevate possono essere ragionevolmente compatibili con il calcare Alberese dell' area del Chianti. La calce prodotta con il calcare Alberese, conosciuta anticamente nel territorio senese come calce "balzana" o "albazzana", veniva utilizzata, nelle varietà più ric- che in minerali argillosi, per la produzione di una calce "forte" adatta a costruire in virtù della sua tenacia e resistenza all'umidità (Fratini, et al., 1994).

Per quanto attiene l'aggregato, si evidenzia una prevalenza della frazione silicoclastica e una buona classazione, indice di una certa attenzione nella scelta delle materie prime. I componenti dell'aggregato sono verosimilmente derivati dalle sabbie fluviali del torrente Arbia, vista la breve distanza dal castello. Si tratta infatti di sedimenti relativamente ben sciacquati dalla componente fine, la cui composizione petrografica è compatibile con le formazioni geologiche attraversate dal torrente stesso (Lazzarotto, et al., in stampa). Notoriamente, la scarsità di una frazione argillosa-siltosa rappresenta un elemento favorevole nella fabbricazione di malte ad elevata resistenza.

Infine, riguardo alla tecnica di preparazione dell'impasto, l'abbondanza di grumi di calce non ben amalgamata di grosse dimensioni, la presenza di innumerevoli masse di concrezioni calcaree derivate da addensamento del legante e la scarsa

\begin{tabular}{lccccccccc}
\hline & $J L s-1$ & $\mathrm{Jls}-1$ & $\begin{array}{c}G B W 0 \\
7108\end{array}$ & $\begin{array}{c}\mathrm{GBW} 0 \\
7108\end{array}$ & 1 & 2 & 3 & 4 & 5 \\
& & & & & & & & & \\
$\mathrm{MgO}$ & 5.19 & 5.23 & 5.19 & 5.23 & 1.25 & 0.71 & 2.10 & 0.67 & 0.41 \\
$\mathrm{SiO}_{2}$ & 15.6 & 15.9 & 15.6 & 15.9 & 13.5 & 22.4 & 17.8 & 23.4 & 29.4 \\
$\mathrm{Na}_{2} \mathrm{O}$ & 0.081 & 0.202 & 0.081 & 0.202 & $<0.001$ & 0.105 & $<0.001$ & 0.140 & 0.113 \\
$\mathrm{~K}_{2} \mathrm{O}$ & 0.780 & 0.756 & 0.780 & 0.756 & $<0.001$ & 0.253 & $<0.001$ & 0.251 & 0.530 \\
$\mathrm{CaO}$ & 35.7 & 36.0 & 35.7 & 36.0 & 44.2 & 36.5 & 39.0 & 38.2 & 31.6 \\
$\mathrm{Al}_{2} \mathrm{O}_{3}$ & 5.03 & 5.11 & 5.03 & 5.11 & 3.10 & 3.90 & 3.74 & 4.52 & 4.70 \\
$\mathrm{Fe}_{2} \mathrm{O}_{3}$ & 2.52 & 2.54 & 2.52 & 2.54 & 1.37 & 1.26 & 2.49 & 0.95 & 0.69 \\
$\mathrm{P}_{2} \mathrm{O}_{5}$ & 0.052 & 0.066 & 0.052 & 0.066 & 0.103 & 0.062 & 0.139 & 0.100 & 0.069 \\
$\mathrm{TiO}_{2}$ & 0.327 & 0.297 & 0.327 & 0.297 & 0.090 & 0.054 & 0.091 & 0.057 & 0.047 \\
$\mathrm{MnO}$ & 0.056 & 0.053 & 0.056 & 0.053 & 0.168 & 0.139 & 0.256 & 0.116 & 0.090 \\
$\mathrm{I.I.}$ & & & & & 0.40 & 0.70 & 0.60 & 1.10 & 0.70 \\
\hline
\end{tabular}

Tab. 2. Risultati delle analisi chimiche dei grumi delle malte della torre ottenute con ICP-OES. I dati sono espressi in wt\%. I.I. = indice di idraulicità. È mostrata l'analisi dei materiali usati come standard, dei quali si riportano anche $\mathrm{i}$ valori di riferimento (in corsivo). Le cifre decimali tengono conto della precisione della misura. 
porosità primaria sono elementi che indicano, con un buon grado di affidabilità, l'utilizzo della tecnica di "calce viva spenta in opera", anche detta "a calce calda". Questa tecnica può fornire alla malta ottime caratteristiche meccaniche ed elevata durabilità, grazie al calore che si sviluppa nello spegnimento della calce con temperature che possono raggiungere i $300{ }^{\circ} \mathrm{C}$ circa. Tale calore, sviluppato direttamente in opera, contribuisce in maniera positiva alla fase di adesione $\mathrm{e}$ reazione fra la calce e l'aggregato, e l'idrata- zione porterebbe ad un aumento di volume con conseguente riduzione della porosità (Foster, 2004; Kraus, et al., 1989; Margalha, et al., 2011; Pecchioni, et al., 2008).

Il lavoro intrapreso sulle malte della torre sarà a breve oggetto di estensione alle altre strutture del complesso, al fine di confermare le osservazioni qui riportate e di fare ulteriore chiarezza sulle vicissitudini storiche di questa importante fortificazione.

\section{Bibliography}

Adams, N. (1993). "L'architettura militare di Francesco di Giorgio", in Fiore, F.P.; Tafuri, M., eds., Francesco di Giorgio architetto - Catalogo della mostra (Siena 1993), Electa Ed., Milano, p. 155.

Cammarosano, P.; Passeri, V. (1985). "Repertorio", in I castelli del Senese, Strutture fortificate dell'area senesegrossetana, Electa Ed., Siena, p. 293.

Cantisani, E.; Falabella, A.; Fratini, F.; Pecchioni, E.; Vettori, S.; Antonelli, F.; Giamello, M.; Lezzerini, M. (2018). "Production of the Roman Cement in Italy: characterization of a raw material used in Tuscany between $19^{\text {th }}$ and $20^{\text {th }}$ century and its comparison with a commercialized French stone material", International Journal of Architectural Heritage, 12(6), pp. 1038-1050.

Chiarelli, N.; Miriello, D.; Bianchi, G.; Fichera, G.; Giamello, M.; Turbanti Memmi, I. (2015). "Characterisation of ancient mortars from the S. Niccolò archaeological complex in Montieri (Tuscany - Italy)", Construction and Building Materials, 96, pp. 442-460.

Chironi, G. (1993). “Appendice documentaria”, in Fiore, F.P.; Tafuri, M., eds., Francesco di Giorgio architetto - Catalogo della mostra (Siena 1993), Electa Ed., Milano, pp. 405-406.

Forster, A. (2004). "Hot lime mortars: a current perspective", Journal of Architectural Conservation, 10(3), pp. 7-27.

Franzini, M.; Leoni, L.; Lezzerini, M.; Sartori, F. (2000). "The mortar of the «Leaning Tower» of Pisa: the product of a medieval technique for preparing high-strength mortars", European Journal of Mineralogy, 12, pp. 1151-1163.

Fratini, F.; Giovannini, P.; Manganelli Del Fà, C. (1994). "La Pietra da calce a Firenze: ricerca e caratterizzazione dei materiali per la produzione di «calcina forte» e «calcina dolce»", in Atti del Convegno "Scienza e Beni Culturali $n^{\circ}$ 10: Bilanci e Prospettive”, Bressanone (Italia), 5-8 luglio 1994, pp. 189-199.

Fusai L. (2010). Mille anni di storia attraverso le vicende della famiglia Cerretani Bandinelli Paparoni. Edizioni ETS, Pisa, Italia.

Gabbrielli, F. (1996). "Scheda n. 79 - Cerretaccio", in Gabbrielli, F.; Rotundo, F., eds., Architettura nel Chianti senese, Catalogo di Castelnuovo Berardenga, Provincia di Siena, Siena, pp. 130-131.

Giamello, M.; Columbu, S.; Gabbrielli, F.; Mugnaini, S.; Scala, A. (2017). "Malte 'fortificate': lo studio delle malte di allettamento nei fortini peruzziani delle mura di Siena (Italia)", in González Avilés, A.B., ed., FORTMED 2017. Defensive Architecture of the Mediterranean. XV to XVIII centuries. Proceedings of the International Conference on Modern Age Fortifications of the Mediterranean Coast, Publicacions Universitat d'Alacant, vol. VI, pp. 283-289.

Guasparri, G.; Mugnaini, S.; Scala, A.; Fratini, F. (2006). "Le malte della Rocca di Montemassi: il supporto scientifico per l'intervento di restauro", in Nazzaro, B., ed., La Rocca di Montemassi: la storia, lo scavo, il restauro, Soprintendenza per i Beni Architettonici e per il Paesaggio per le Provincie di Siena e Grosseto, Edizioni Kappa, Roma, pp. 59-68.

Kraus, K.; Wisser, S.; Knofel, D. (1989). "Uber das Loschen von Kalk in de 18 Jahrunderts: Literaturswertung und Laborversuche", Arbeitsblatter fur Restauratoren, 1, pp. 206-221 
Lazzarotto, A.; Costantini, A.; Sandrelli, F.; Brogi, A.; Foresi, L.M. (in stampa). Carta Geologica d'Italia alla scala 1:50.000, Foglio 297 “Asciano”, Servizio Geologico d'Italia, Roma.

Lezzerini, M.; Ramacciotti, M.; Cantini, F.; Fatighenti, F.; Antonelli, F.; Pecchioni, E.; Fratini, F.; Cantisani, E.; Giamello, M. (2017). "Archaeometric study of natural hydraulic mortars: the case of the Late Roman Villa dell'Oratorio (Florence, Italy)", Archaeological and Anthropological Sciences, 9(4), pp. 603-615.

Marchetti, F. (2016). "I castelli descritti dalle vestigia: Orgiale, Le Stinche, Monternano, Cerreto Ciampoli”, in Pellegrini, E., ed., Fortificare con arte. Torri, casseri e fortezze nel Chianti, Betti Ed., Siena, pp. 122-135.

Margalha, G.; Veiga, R.; Santos Silva, A.; Brito, J. de. (2011). "Traditional methods of mortar preparation: the hot lime mix method", Cement \& Concrete Composites, 33, pp. 796-804.

Martini, F. di Giorgio. (1967). Trattati di Architettura, Ingegneria e Arte Militare, a cura di Corrado Maltese, Ed. Il Polifilo, Milano, Italia.

Moropoulou, A.; Bakolas, A.; Bisbikou, K. (2000). "Investigation of the technology of historic mortars", Journal of Cultural Heritage, 1(1), pp. 45-58.

Parenti, R.; Doglioni, F. (1993). "Murature a sacco o murature a nucleo in calcestruzzo? Precisazioni preliminari desunte dall'osservazione di sezioni murarie", in Biscontin, G.; Mietto, D., eds., "Calcestruzzi antichi e moderni: storia, cultura e tecnologia”, Atti del convegno di studi (Bressanone 6-9 luglio 1993), Scienza e beni culturali IX, Libreria Progetto Editore, Padova, pp. 150-151.

Passeri, V. (2002). Documenti per la storia delle località della provincia di Siena, Edizioni Cantagalli, Siena, Italia, pp. 83-84.

Pecchioni, E.; Fratini, F.; Cantisani, E. (2006). "The ancient mortars, an attestation of the material culture: the case of Florence", Periodico di Mineralogia, 75, pp. 303-324.

Pecchioni, E.; Fratini, F.; Cantisani, E. (2008). Le malte antiche e moderne tra tradizione ed innovazione, Pàtron Editore, Bologna, pp.44-46.

Riccardi, M.; Lezzerini, M.; Carò, F.; Franzini, M.; Messiga, B. (2007). "Microtextural and microchemical studies of hydraulic ancient mortars: two analytical approaches to understand pre-industrial technology processes", Journal of Cultural Heritage, 8, pp. 350-360.

Valenti, M. (1995). "Scheda n. 50 - Cerretaccio (Cerreto Ciampoli)", in Valenti, M., ed., Carta archeologica della provincia di Siena, vol. I, Il Chianti senese, Nuova Immagine Editrice, Siena, pp. 221-222. 